\title{
EFEITOS DOS MÉTODOS PIRÂMIDE CRESCENTE E DECRESCENTE NO NÚMERO DE REPETIÇÕES DO TREINAMENTO DE FORÇA EM MULHERES JOVENS
}

\section{EFFECTS OF METHODS PYRAMID CRESCENT AND DECLINING IN NUMBER OF STRENGTH TRAINING REPS IN YOUNG WOMEN}

\author{
Lucas Vieira Assunção, ${ }_{1}^{1}$ Jefferson Fernando Coelho Rodrigues Júnior, ${ }^{1}$ Igor Ferreira Nunes, ${ }^{1}$ Vânia Silva \\ Macedo Orsano ${ }^{1}$ \\ ${ }^{1}$ Universidade Federal do Piauí/Teresina/Brasil.
}

Autor correspondente: Jefferson Fernando Coelho Rodrigues Júnior e-mail: jefferssonfernando@ hotmail.com

\section{EDITORES}

Thiago Gomes Heck

Adriane Cristina Bernat Kolankiewicz (Unijuí-Brasil)

\section{EDITORES DE ÁREA}

Educação \& Saúde

Eva Teresinha de Oliveira Boff

(Unijuí-Brasil)

Fisioterapia \& Saúde

Eliane Roseli Winkelmann

(Unijuí-Brasil)

Ciências Farmacêuticas \& Saúde

Marilei Uecker Pletsch (Unijuí-Brasil)

\section{Nutrição \& Saúde}

Lígia Beatriz Bento Franz

(Unijuí-Brasil)

Nadia Oliveira

Ingrid Perry

Enfermagem e suas contribuições para a prática

Adriane Cristina Kolankiewicz

(Unijuí-Brasil)

Crhis de Brum

Neila de Souza

\section{Exercício Físico \& Saúde}

Thiago Gomes Heck

(Unijuí-Brasil)

Anderson Zampier Ulbrich

(UFPR)

Editora Unijuí

Universidade Regional do Noroeste do

Estado do Rio Grande do Sul (Unijuí)

\section{RESUMO}

Introdução: o Treinamento de Força (TF) vem sendo muito utilizado em razão dos benefícios proporcionados aos praticantes, além de apresentarem uma variedade de métodos com diferentes finalidades. Objetivo: comparar o efeito dos métodos pirâmide crescente e decrescente sobre o número total de repetições máximas (RM) produzidas em três séries no exercício flexora sentada em uma sessão de treinamento de força (TF). Metodologia: participaram 10 mulheres jovens $(24,5 \pm 8,5$ anos; $53,9 \pm 14,1 \mathrm{Kg} ; 158 \pm 7 \mathrm{~cm})$, com prática em TF de no mínimo seis meses. Os dados foram obtidos em quatro dias não consecutivos ao longo de duas semanas: Para a pirâmide crescente foram adotadas três séries com intensidades de $70 \%, 80 \%$ e $90 \%$ da carga de 1RM, e para a pirâmide decrescente a ordem das intensidades foi inversa, sendo o intervalo entre as séries de três minutos. Para analisar a diferença entre os métodos utilizou-se o teste t student com critério de significância de $5 \%$. Resultados: não foram encontradas diferenças significativas entre o número total de RM realizadas nos dois protocolos experimentais, no entanto para as intensidades de $90 \%$ e $80 \%$ foi realizado um maior número de RM no protocolo de pirâmide decrescente. Já na pirâmide crescente houve maior número de RM na intensidade de $70 \%$ em relação à mesma intensidade no método pirâmide decrescente. Conclusão: o método pirâmide decrescente seria proveitoso para adaptação na transição de treinos tensionais para metabólicos e, quando o treino for com aumento progressivo de volume da carga, a pirâmide crescente se fez mais eficaz.

Palavras-chave: Treinamento de força. Musculação. Hipertrofia.

Submetido em: 13/3/2016

Aceito em: $31 / 10 / 2016$ 


\section{Revista}

\section{Contexto}

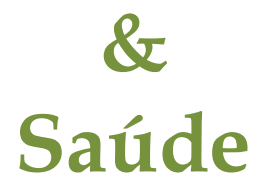

Volume 16

Número 31

2016

ISSN 2176-7114

A Revista Contexto \& Saúde é um periódico do Departamento de Ciências da Vida da Universidade Regional do Noroeste do Estado do Rio Grande do Sul (Unijuí). É um periódico semestral que tem por objetivo a divulgação da produção técnico-científica de temas relacionados à área de Ciências da Saúde.

O escopo da revista abrange a divulgação de resultados de pesquisa que contemplem avanços no processo saúde-doençacuidado e no conhecimento e aplicabilidade de novos processos químicos e biológicos em saúde.

Neste periódico, entende-se que a publicação de estudos com os aspectos epidemiológicos, assistenciais e educacionais em saúde, experimentais e aplicados é uma forma a subsidiar e qualificar a atenção à saúde de modo interdisciplinar.

\begin{abstract}
Introduction: Strength Training (ST) has been broadly used due to its benefits to the subjects. It presents many structures and methods, with different objectives. Objective: To compare the effects of the ascending and descending pyramid method in the net number of maximum repetitions (MR) produced in three series in the exercise curl machine in a strength training session. Methods: 10 young women $(24,5 \pm 8,5$ years old; $53,9 \pm 14,1 \mathrm{~kg} ; 158 \pm 7 \mathrm{~cm})$ performed the strength training for at least six months. The data was obtained in four nonconsecutive days during two weeks. For the ascending pyramid, it was performed three series with intensities of $70 \%, 80 \%$ and $90 \%$ of the load of $1 \mathrm{MR}$, and for the descending pyramid the order of the intensity was the inverse, with an interval between series of three minutes. In order to analyse the difference between the methods, it was used the Student t-test with significance level of 5\%. Results: There was no significant difference between the net numbers of maximum repetitions performed in both protocols. However, in the intensities of $90 \%$ and $80 \%$, it was performed a greater number of maximum repetitions in the descending pyramid protocol. In the ascending pyramid protocol, on the other hand, there was a greater number of MR in the intensity of $70 \%$ compared to the descending pyramid method in the same intensity. Conclusion: Descending pyramid method would be beneficial for the adaptation in the transition of tensional trainings to the metabolic trainings and when he training is with progressive increasing of load volume, ascending pyramid method is pointed out to be more efficient.
\end{abstract}

Keywords: Strength training. Bodybuilding. Hypertrophy. 


\section{INTRODUÇÃO}

O treinamento de força ou treinamento resistido é uma forma especializada de melhorar o desempenho, saúde e aptidão física, que se constitui de uma variedade de exercícios contra uma resistência, além de contar com uma variedade de métodos de treinamento (FAIGENBAUM et al., 2009), sendo indicado para diferentes populações para a melhoria da aptidão física, aumento da velocidade, força, massa muscular, potência, desempenho motor, resistência de força, coordenação e equilíbrio (TIGGEMANN, 2013).

Em virtude de todas as adaptações e benefícios proporcionados pelo treinamento de força, vários protocolos, métodos e sistemas de treinamento foram criados para otimizar o processo de hipertrofia, por meio da manipulação e combinação das variáveis de treinamento como número de repetições, carga, número de séries, intervalo de recuperação, entre outros (WEINECK, 2003; KENNEY; WILMORE; COSTIL, 2013; SALLES et al., 2014).

Os métodos de treinamento de força proporcionam o crescimento das fibras musculares do tipo II, responsáveis pelo aumento de força e potência. Essas adaptações ao músculo esquelético podem sofrer variações na quantidade, comprimento longitudinal e volume da secção transversa, ocasionado por microlesões provocadas por esse tipo de treinamento, seguido por uma regeneração muscular por participação das células satélites (BEZERRA NETO; NAVARRO, 2013).

Um método que tem sido bastante utilizado como estratégia para o aumento da força e hipertrofia é o da pirâmide, que se fundamenta na correlação volume versus intensidade de treinamento, quando a cada grupo de determinado exercício ocorre a diminuição ou o aumento do número de repetições realizadas e o simultâneo aumento ou diminuição do peso. Sua aplicação se dá na manipulação da carga de forma crescente ou decrescente em relação à intensidade. Como consequência ocorrem modificações no número de repetições realizadas (MAZINI FILHO et al., 2015).

Um estudo recente (MAZINI FILHO et al., 2015), comparando os métodos crescente e decrescente, observou que as duas formas se mostraram efetivas para o treinamento, visando o ganho de força em pequenos grupamentos e sugerindo novos estudos entre pequenos e grandes grupamentos.

Outro estudo (SALLES et al., 2008) comparou o sistema piramidal crescente e decrescente sobre o número total de repetições máximas em uma sessão de treino de força, em que não foi encontrada diferença entre as repetições máximas totais realizadas nos dois sistemas de treino. Este estudo restringiu a amostra a homens experientes.

Em razão da grande utilização deste método, torna-se importante estabelecer um maior embasamento científico acerca de sua aplicabilidade e eficiência em diferentes populações, com e sem experiência em treino de força muscular e de ambos os sexos, quanto às formas, Crescente (Delorme) e Decrescente (Oxford), no treinamento de força. Assim, este estudo teve como objetivo comparar o efeito dos métodos pirâmide crescente (Delorme) e decrescente (Oxford) sobre o número total de RM, utilizando diferentes intensidades de treinamento.

\section{METODOLOGIA}

\section{Tipo de Estudo}

Trata-se de uma pesquisa quantitativa, pois utiliza o auxílio matemático (estatístico) para chegar a um resultado das causas de um fenômeno e suas possíveis relações (GERHARDT; SILVEIRA, 2009), e experimental, definido por Thomas, Nelson e Stephen (2009) como pesquisa adequada para avaliar um sistema ou método por 
possuir um grande controle sobre a amostra e fatores externos que podem influenciar os resultados. Será empregado o método dedutivo que, segundo Pereira (1998), se baseia em um conhecimento para criar hipóteses sendo submetidas a testes a fim de se chegar a uma conclusão.

\section{Sujeitos do Estudo}

Participaram do experimento dez indivíduos (mulheres jovens; 24,5 \pm 8,5 anos) com experiência mínima de seis meses no treinamento de força, com frequência mínima de três vezes por semana. Foram excluídos do estudo indivíduos usuários de medicamentos, sejam estes em prol da saúde ou em benefício do desempenho (recursos ergogênicos), e indivíduos que apresentaram qualquer tipo de limitação articular ou problemas osteomioarticulares que pudessem influenciar a realização do exercício proposto.

\section{Critérios de Inclusão e Exclusão}

Foram incluídos no estudo apenas praticantes de musculação que treinavam três vezes por semana e, no mínimo, há seis meses.

Foram excluídos do estudo participantes que apresentassem alguma doença crônico-degenerativa, fossem tabagistas, tivessem alguma disfunção no histórico de saúde ou outro problema que pudesse comprometer a integridade física e a execução da pesquisa, e não poderiam apresentar problemas ortopédicos que limitassem a participação na sessão de treino.

\section{Aspectos Éticos}

Todos os procedimentos foram realizados segundo princípios éticos estabelecidos na Resolução $\mathrm{n}^{\circ} 466$, de 12 de dezembro de 2012 pelo Conselho Nacional de Saúde. O projeto foi submetido ao Comitê de Ética da Universidade Federal do Piauí - Ufpi - e aprovado conforme número 48243815.0.0000.5214.

Todos os participantes assinaram o Termo de Consentimento Livre e Esclarecido. Foram expostos todos os benefícios e o mínimo de riscos possíveis a eles, deixando claro que todos teriam o livre-arbítrio de participar ou não da pesquisa, ou abandonar a mesma em qualquer momento que achassem necessário, sendo garantido que não seriam penalizadas caso isso ocorresse.

Todos tiveram acesso ao projeto, para que, assim, pudessem saber de tudo o que iria acontecer, quais as propostas, objetivos, metodologias, cronograma, bem como os seus direitos. O trabalho obedeceu à metodologia adequada para evitar qualquer dano aos participantes, com total confidencialidade e privacidade para a proteção da imagem e a não utilização das informações adquiridas em prejuízo ou degradação dos mesmos.

\section{Instrumentos para coleta de dados}

Para a coleta dos dados foram utilizados os materiais a seguir: balança antropométrica e estadiômetro (Welmy), para aferir o peso e a estatura respectivamente dos indivíduos participantes do estudo, e um equipamento flexora sentada (Gervasport), onde foram executados os testes e retestes de uma repetição máxima, e os exercícios pelos métodos da pirâmide crescente (Delorme) e decrescente (Oxford). 


\section{Procedimentos para coleta de dados}

Antes da coleta dos dados as voluntárias responderam ao questionário PAR-Q, elaborado com o objetivo de identificar a necessidade de avaliação clínica e médica antes do início de uma atividade física. Também assinaram um termo de participação consentida, conforme Resolução 466/2012 do Conselho Nacional de Saúde.

Os dados foram coletados em quatro dias não consecutivos ao longo de duas semanas: no primeiro dia foi realizado o teste de uma repetição máxima no equipamento flexora sentada, 72 horas após o teste de 1RM foi feito o reteste do mesmo, no aparelho já citado, para a obtenção da reprodutibilidade da carga no teste 1RM. Este procedimento teve a finalidade de disponibilizar a informação acerca da carga máxima suportada por indivíduo em uma repetição, a qual, posteriormente, foi manipulada mediante porcentuais para definir a carga de cada série na pirâmide crescente (Delorme) e decrescente (Oxford) (utilizadas no exercício que será explanado a seguir); nos dias 3 e 4 (com intervalo de 48 horas entre eles) foi realizado o protocolo de treinamento das pirâmides crescente (Delorme) ou decrescente (Oxford) com entrada alternada, quando o grupo 1 iniciou o exercício com a pirâmide crescente (Delorme) e, no segundo dia, realizou-se a pirâmide decrescente (Oxford), e o grupo 2 deu início ao teste com a pirâmide decrescente (Oxford) e no segundo dia do procedimento fez a pirâmide crescente (Delorme). Para a pirâmide crescente (Delorme) foram adotadas três séries com intensidades de $70 \%, 80 \%$ e $90 \%$ da carga de 1RM, e para a pirâmide decrescente (Oxford) a ordem das intensidades foi inversa. $\mathrm{O}$ intervalo entre as séries em todas as intensidades verificadas foi determinado em três minutos.

\section{Teste de 1RM}

Para obtenção da carga de 1RM executou-se o procedimento a seguir: as avaliadas realizaram um aquecimento específico no equipamento flexora sentada, com carga leve para a realização de 12 repetições de forma confortável. Após intervalo de dois minutos adicionou-se carga e as avaliadas foram instruídas a realizarem uma repetição. Na medida em que a participante foi conseguindo vencer a resistência, a carga era incrementada progressivamente de 7 a 14 quilogramas por, no máximo, três tentativas, com intervalos de três minutos entre as tentativas (SALLES et al., 2008). Em todas as séries o tempo da fase excêntrica e concêntrica foi controlado por um avaliador portando um cronômetro.

Após a aquisição da carga máxima no teste de 1RM, as participantes tiveram um intervalo de descanso por 72 horas e foram reavaliadas para obtenção da reprodutibilidade da carga no teste 1RM (segundo dia de teste). Declarou-se como carga para 1RM a maior carga estabelecida em ambos os dias sem que houvesse execução incorreta da técnica.

Tendo em vista diminuir a margem de erro nos testes de 1RM, foram aderidas as seguintes estratégias. 1) instruções padronizadas foram fornecidas antes do teste, de modo que a avaliada estivesse a par de toda a rotina que envolveu a coleta de dados; 2) a avaliada foi informada a respeito da técnica de execução do exercício; 3 ) o avaliador esteve vigilante quanto à posição adotada pela praticante no momento da aferição, pois pequenas variações no posicionamento das articulações envolvidas no movimento poderiam mobilizar outros músculos, levando a análises errôneas dos dados obtidos; 4) incentivos verbais foram efetuados a fim de manter alto o nível de estimulação.

Foram obedecidas as seguintes etapas de execução do exercício: posição inicial e fase concêntrica. Posição inicial - sentado no equipamento com os joelhos semiflexionados, braços ao longo do corpo (paralelos ao trono) e mãos segurando nos pegadores laterais; Fase concêntrica - a partir da posição inicial foi realizada a flexão dos joelhos até formar uma angulação de $90^{\circ}$ entre coxa e perna. 


\section{Protocolo da sessão de treinamento e de coleta}

O protocolo de treinamento foi realizado 72 horas após o último dia de teste de 1RM, e consistiu de duas sessões para cada voluntária, com 48 horas de intervalo entre as sessões. Para determinar a ordem de realização dos protocolos, as voluntárias foram divididas por meio de um sorteio em dois grupos usando delineamento alternado, com entrada intercalada no grupo um (G1) e dois (G2). O G1 na primeira sessão de treinamento realizou a pirâmide crescente (Delorme), que consistiu em três séries com intensidade de $70 \%, 80 \%$ e $90 \%$ de 1RM respectivamente, e, após o intervalo de 48 horas, na segunda sessão de treinamento foi realizada a pirâmide decrescente (Oxford) na ordem inversa. Já o G2 realizou a pirâmide decrescente (Oxford) na primeira sessão de treinamento e a pirâmide crescente (Delorme) na segunda sessão.

As participantes realizaram o exercício até a falha muscular concêntrica, estabelecendo-se dois segundos para cada fase de contração, totalizando quatro segundos em cada repetição (movimento completo) (REIS et al., 2015; ASSUNÇÃO et al., 2007) sem variar o padrão do movimento; foram adotados três minutos de recuperação entre as séries. O número de RM, acrescentando a repetição em que ocorreu a falha concêntrica, foi catalogado ao final de cada uma das três séries em ambas as sequências. Como aquecimento, foram efetuadas 12 repetições com $40 \%$ a $50 \%$ da carga de $1 \mathrm{RM}$ nos dois segmentos.

\section{Tratamento estatístico}

O processamento dos dados e a análise estatística foram realizados por meio do programa SPSS®, versão 18.0. As variáveis quantitativas foram apresentadas por estatística descritiva: média e desvio-padrão. Primeiramente foi aplicado o teste de Shapiro-Wilk para avaliar a normalidade das variáveis quantitativas e, para analisar as pirâmides crescente (Delorme) e decrescente (Oxford), utilizou-se o teste t student com critério de significância estatística estabelecido de $5 \%$.

\section{RESULTADOS E DISCUSSÃO}

A partir da apresentação dos dados em média e desvio-padrão, não foram encontradas diferenças significativas entre o número total de RM realizadas nos dois protocolos experimentais, conforme Gráfico 1. Porém, foram obtidas diferenças entre métodos para os números de RMs realizadas com a mesma intensidade, evidenciados na Tabela 1. 
Gráfico 1 - Número total de RMs produzidas no protocolo de pirâmide crescente (Delorme) e decrescente (Oxford) (média \pm desvio padrão)

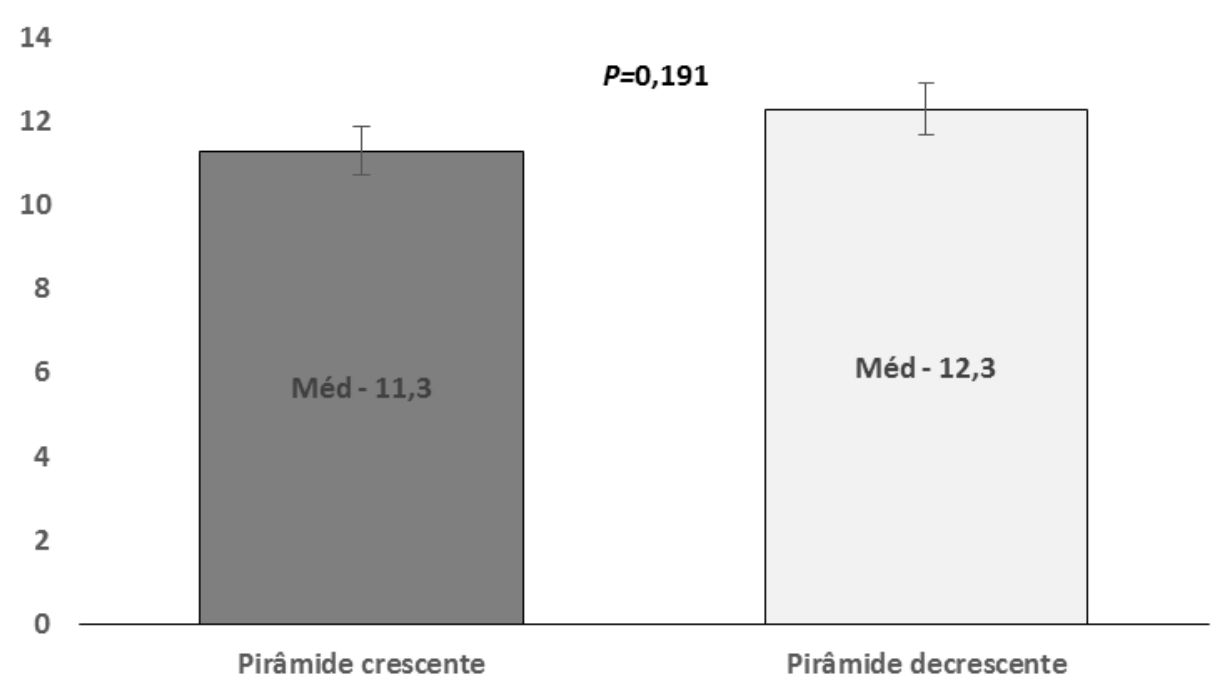

Tabela 1 - Número total de RMs produzidas no protocolo de pirâmide crescente (Delorme) e decrescente (Oxford) (média e desvio-padrão)

\begin{tabular}{llll}
\hline & \multicolumn{2}{c}{ Método } & \multirow{2}{*}{$\boldsymbol{P}^{*}$} \\
\cline { 2 - 3 } Série & \multicolumn{1}{c}{ Pirâmide crescente (Delorme) } & Pirâmide decrescente (Oxford) & $\mathbf{0 , 0 0 6 *}$ \\
\hline $\mathbf{7 0 \%}$ RM & $19,6 \pm 4,8$ & $16,1 \pm 2,5$ & $\mathbf{0 , 0 0 2 \#}$ \\
$\mathbf{8 0 \%}$ RM & $10,3 \pm 3,4$ & $12,8 \pm 2,9$ & $<\mathbf{0 , 0 0 1 \#}$ \\
$\mathbf{9 0 \%}$ RM & $4,0 \pm 3,1$ & $7,9 \pm 2,6$ & \\
\hline
\end{tabular}

*p $\leq 0,05$ em relação às intensidades $70 \%, 80 \%$ e $90 \%$ \#Estatisticamente significante $\mathbf{p}<0,05$

Para todas as intensidades de $1 \mathrm{RM}$ da carga foram apresentadas diferenças significativas, as participantes realizaram um maior número de repetições no protocolo de pirâmide decrescente (Oxford) quando comparado às mesmas intensidades na pirâmide crescente (Delorme), exceto na intensidade de $70 \%$ de 1 RM. Isso se explica pelo fato de que a realização preliminar de um grande número de contrações feitas nas intensidades de $70 \%$ de 1RM na pirâmide crescente (Delorme) prejudicou o mecanismo de contração-relaxamento por múltiplos fatores; como a liberação de cálcio pelo retículo sarcoplasmático e diminuição da excitabilidade da membrana celular, ocasionando um menor recrutamento de unidades motoras e restringindo a eficácia de conceber força (CHIN; BALNAVE; ALLEN, 1997; GREEN, 1997; MAZINI FILHO et al., 2015).

Na intensidade de $70 \%$ da carga de 1RM, houve um maior número de RM no método de pirâmide crescente (Delorme) em relação à mesma intensidade no método pirâmide decrescente (Oxford). Essa queda nos números de repetições máximas, observadas em diferentes intensidades nos dois métodos, pode estar associada também ao intervalo aderido entre as séries definido em três minutos. Intervalos de três minutos ou menos podem acarretar em diminuição significativas do número de repetições máximas durante o prosseguimento das séries, mesmo sem acréscimo de carga (SALLES et al., 2008).

Realizar exercícios que iniciem com séries de 19 repetições e terminem com séries de 4 repetições, como ocorreu nas médias das séries da pirâmide crescente (Delorme) realizada nesse estudo, não seria recomendado, 
pois as alterações metabólicas geradas pelas repetições altas não foram mantidas devido a diminuição do número de repetições nas séries seguintes. Isso ocorreu devido à fadiga prévia gerada pelas repetições altas realizadas na primeira série da pirâmide crescente (Delorme), diminuindo assim, a magnitude dos estímulos tensionais na última série deste método (MAZINI FILHO et al., 2015).

Já na pirâmide decrescente (Oxford) as repetições das séries de $80 \%$ e $90 \%$ da carga de 1RM se mantiveram dentro de níveis adequados para proporcionar adaptações hipertróficas, isto é, com repetições médias de aproximadamente 12 e 8 , e na série de $70 \%$ da carga de $1 \mathrm{RM}$ foi realizada a média de 16 repetições (possuindo características metabólicas), iniciadas com estresse bioquímico mais acentuado, o que poderia ser benéfico para hipertrofia (MAZINI FILHO et al., 2015).

O método pirâmide crescente (Delorme) se mostra eficaz como forma de "aquecimento" sem o comprometimento do número total de repetições, atualmente é empregada com repetições máximas ou próximas das máximas e com uma gradativa redução das repetições e aumento das cargas, mas não há uma preocupação aparente de não provocar fadiga (FISH et al., 2003). O presente experimento apresentou, porém, queda no rendimento conforme se progredia para séries de maior intensidade. Por esse motivo pode existir um prejuízo no ganho de força em longo prazo (MCNORRIS; ELKINS, 1954; SALLES et al., 2008), pelo fato de ela deter um alto número de repetições nas primeiras séries, que acabam por compensar o alto declínio no número de repetição na última série, não comprometendo, assim, o número total de repetições máximas (SILVA et al., 2010).

Um estudo feito por Gentil (2005) mostra que, quando utilizado o método pirâmide crescente (Delorme) há dificuldades durante a realização da terceira série pela fadiga do músculo resultante das séries anteriores. Tal consideração levou à utilização do método pirâmide decrescente (Oxford), que é um método inverso ao Delorme, no qual a aplicação de cargas mais altas no início da série desfrutaria do estado neural para oferecer estímulos tensionais, e as séries seguintes, que eventualmente tivessem características metabólicas, seriam iniciadas com estresse bioquímico mais enfatizado, o que poderia ser favorável para a hipertrofia. De fato, no estudo em questão foi averiguada uma menor discrepância no número de repetições entre as séries da pirâmide decrescente (Oxford), o que faz dela um método mais propício do que a pirâmide crescente (Delorme), na possibilidade de ocasionar hipertrofia.

Diante dos resultados as participantes apresentaram um maior número de repetições máximas nas intensidades de 80 e $90 \%$ no protocolo de pirâmide decrescente (Oxford) quando comparado às mesmas intensidades na pirâmide crescente (Delorme). Isso devido ao grupamento muscular estar descansado em pirâmides decrescentes (Oxford) nas séries iniciais suportando mais cargas. Levando a inferir que o método pirâmide decrescente (Oxford) seria proveitoso para adaptação na transição de treinos tensionais para metabólicos em praticantes de treinamento de força acostumados a treinar com repetições altas por muito tempo (MAZINI FILHO, 2015).

Por outro lado, os métodos metabólicos incitam a hipertrofia por meio, primordialmente, de modificações nas condições metabólicas locais. Estes métodos têm como vantagem não necessitar da exposição das articulações a altas cargas, podendo ser usados em alunos lesionados, iniciantes e em estágios que se deseje descansar ossos e tendões (GENTIL, 2005).

Pela limitação dos métodos, diversos fatores podem ter interferido nos resultados alcançados, podendo-se frisar: a estabilização postural, a velocidade de execução, a redução da atividade do antagonista, a aprendizagem na coordenação, o tipo de fibra muscular envolvida e a intensidade das cargas aplicadas. Novos experimentos 
utilizando outros exercícios e intensidades devem ser realizados para verificar o efeito desses métodos de treinamento.

\section{CONCLUSÃO}

Os resultados adquiridos no presente estudo mostraram que os métodos pirâmide crescente (Delorme) e decrescente (Oxford) não expuseram efeitos agudos divergentes sobre o volume total de RM no exercício flexora sentada para as intensidades adotadas. Para as intensidades de $90 \%$ e $80 \%$ da carga de 1 RM, no entanto, as participantes apresentaram valores significativos à realização de um maior número de RM no protocolo de pirâmide decrescente (Oxford) quando comparado às mesmas intensidades na pirâmide crescente (Delorme). Já na pirâmide crescente (Delorme) houve maior número de repetições na intensidade de $70 \%$ da carga de $1 \mathrm{RM}$ em relação à mesma intensidade no método pirâmide decrescente (Oxford).

\section{REFERENCIAS}

ASSUNÇÃO, W. D. et al. Respostas cardiovasculares agudas no treinamento de força conduzido em exercícios para grandes e pequenos grupamentos musculares. Rev Bras Med Esporte, 13.2, p. 118-22, 2007.

BEZERRA NETO, J. C.; NAVARRO, F. Influência do treinamento de força em idosos na modulação das células satélites: uma revisão sistemática. Revista Brasileira de Prescrição e Fisiologia do Exercício (RBPFEX), 7.42, 2013.

CHIN, E. R.; BALNAVE, C. D.; ALLEN, D. G. Role of intracellular calcium and metabolites in low-frequency fatigue of mouse skeletal muscle. American Journal of Physiology, v. 272, n. 2, p. C550-C559, 1997.

FAIGENBAUM, A. D. et al. Youth resistance training: Updated position statement paper from the national strength and conditioning association. J Strength Cond Res, 23: S60-S79, 2009.

FISH, David E. et al. Optimal resistance training: comparison of DeLorme with Oxford techniques. American journal of physical medicine \& rehabilitation, v. 82, n. 12, p. 903-909, 2003.

GENTIL, P. Bases científicas do treinamento de hipertrofia. 1. ed. Rio de Janeiro: Sprint, 2005. 192 p.

GERHARDT, T. E.; SILVEIRA, D. T. Métodos de pesquisa. Porto Alegre: Editora da UFRGS, 2009.

GREEN, H. J. Mechanisms of muscle fatigue in intense exercise. Journal of Sports Sciences, v. 15, n. 3, p. 247-256, 1997.

KENNEY, W. L.; WILMORE, J. H.; COSTIL, D. L. Fisiologia do esporte e do exercício. 5. ed. São Paulo: Manole, 2013.

MAZINI FILHO, Mauro Lúcio et al. Comparação dos métodos pirâmide crescente e decrescente no aumento da força muscular. RBPFEX-Revista Brasileira de Prescrição e Fisiologia do Exercício, 9.53, 240-249, 2015.

MCNORRIS, R. O.; ELKINS, E. C. A. Study of production and evaluation of muscular hypertrophy. Archives of Physical Medicine Rehabilitation, vol. 35, n. 1, 1954.

PEREIRA, B. As limitações do método científico: implicações para a educação física. Revista Paulista de Educação Física, São Paulo, 12.2 (1998), p. 228-48, 1998.

REIS, J. P. C. et al. Efeito do Treinamento Resistido com Diferentes Intensidades na Pressão Arterial em Hipertensos. Int J Cardiovasc Sci, 1.1, p. 25-34, 2015.

SALLES, B. F. et al. Efeito dos métodos pirâmide crescente e pirâmide decrescente no número de repetições do treinamento de força. Revista Eletrônica da Escola de Educação Física e Desportos, UFRJ, v. 4. n. 1, jan./jun. 2008. 
SILVA, D. P. et al. Resposta crônica da pressão arterial sistólica em diferentes métodos de treinamento de força: Delorme versus Oxford. Perspectivas OnLine, 2007-2010, 3.12 (2014).

. Comparison of DeLorme with Oxford resistance training techniques: effects of training on muscle damage markers. Biology of Sport, v. 27, p. 77, 2010.

THOMAS, J. R.; NELSON, J. K.; STEPHEN J. S. Métodos de pesquisa em atividade física. Porto Alegre: Artmed Editora, 2009.

TIGGEMANN, C. L. Comparação entre métodos de determinação da carga e de velocidade de execução do treinamento de força nas adaptações neuromusculares e no desempenho de capacidades funcionais em mulheres idosas: ensaio clínico randomizado. Lume. UFRGS, 2013.

WEINECK, J. Atividade física e esporte para que? São Paulo: Manole, 2003. 